\title{
Lyotropic Colloidal and Macromolecular Liquid Crystals
}

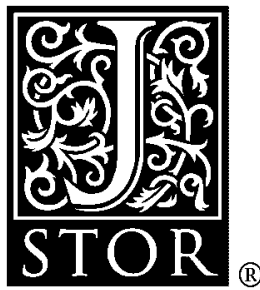

\author{
H. N. W. Lekkerkerker; G. J. Vroege
}

Philosophical Transactions: Physical Sciences and Engineering, Vol. 344, No. 1672, Understanding Self-Assembly and Organization in Liquid Crystals. (Aug. 16, 1993), pp. 419-440.

Stable URL:

http://links.jstor.org/sici?sici=0962-8428\%2819930816\%29344\%3A1672\%3C419\%3ALCAMLC\%3E2.0.CO\%3B2-1

Philosophical Transactions: Physical Sciences and Engineering is currently published by The Royal Society.

Your use of the JSTOR archive indicates your acceptance of JSTOR's Terms and Conditions of Use, available at http://www.jstor.org/about/terms.html. JSTOR's Terms and Conditions of Use provides, in part, that unless you have obtained prior permission, you may not download an entire issue of a journal or multiple copies of articles, and you may use content in the JSTOR archive only for your personal, non-commercial use.

Please contact the publisher regarding any further use of this work. Publisher contact information may be obtained at http://www.jstor.org/journals/rsl.html.

Each copy of any part of a JSTOR transmission must contain the same copyright notice that appears on the screen or printed page of such transmission.

The JSTOR Archive is a trusted digital repository providing for long-term preservation and access to leading academic journals and scholarly literature from around the world. The Archive is supported by libraries, scholarly societies, publishers, and foundations. It is an initiative of JSTOR, a not-for-profit organization with a mission to help the scholarly community take advantage of advances in technology. For more information regarding JSTOR, please contact support@jstor.org. 


\title{
Lyotropic colloidal and macromolecular liquid crystals
}

\author{
By H. N. W. LEKKERKERKER AND G. J. VROEGE \\ Van 't Hoff Laboratory, Utrecht University, Padualaan 8, 3584 CH Utrecht, \\ The Netherlands
}

We review the theory of the isotropic-nematic phase transition for solutions of thin hard rods and semi-flexible chain molecules along with the extensions to polydisperse systems and soft interactions. The occurrence of more highly ordered liquid crystal phases (smectic, columnar) in concentrated solutions of colloids and macromolecules is discussed briefly. Experimental results for a number of carefully studied uncharged and charged colloids and macromolecules are compared to theoretical results.

\section{Introduction}

In this paper we present a brief overview of theory and experiments on liquid crystal phases which appear in solutions of elongated colloidal particles or stiff polymers. In the 40s Onsager $(1942,1949)$ presented his theory of the isotropic-nematic transition of a monodisperse system of thin hard rods, which was published in his seminal paper of 1949. Onsager showed that the isotropic-nematic transition can be explained on the basis of merely two-particle interactions represented by the second virial term in an expansion of the free energy of the system. Although the direct relevance of the Onsager theory for the explanation of experiments is rather limited, being restricted to low volume fractions of perfectly rigid very long rods, its extensions by introducing polydispersity, electrostatic interactions and semi-flexibility and developments for higher volume fractions have been applied with good results to polymer and colloidal solutions.

We describe the Onsager theory in some detail in $\S 2$. The effects of polydispersity, electrostatic and attractive interactions are described in $\$ \S 3-5$. For the applications of virial theories a strong impediment has been its limitation to rigid particles. This has changed with the extension to semi-flexible (wormlike chain) polymers by Khokhlov \& Semenov $(1981,1982)$ which is treated in $\$ 6$, along with alternative formulations which provide a clear physical insight. Finally in $\S 7$ we compare the theory to experimental results on a number of selected systems representative for neutral and charged rigid rod-like colloids and semi-flexible macromolecules.

\section{Liquid crystal phase transitions in dispersions of thin hard rods}

(a) The isotropic-nematic transition

As alluded to in the introduction our starting point in this review is Onsager's theory for the isotropic-nematic phase transition in dispersions of thin hard rods. This theory is based upon the following second virial approximation for the free energy of $N$ rigid hard rods with length $L$ and diameter $D(L / D \gg 1)$ in a dispersion with volume $V$ :

$$
\Delta F[f] / N k T=\text { const. }+\ln c+\sigma[f]+c \rho[f] .
$$


Here $f(\Omega)$ is the orientation distribution function which gives the probability of finding a rod with an orientation characterized by solid angle $\Omega$. This distribution function must be normalized:

$$
\int f(\Omega) \mathrm{d} \Omega=1 .
$$

The (dimensionless) concentration $c$ is defined as follows:

$$
c=\frac{1}{4} \pi L^{2} D N / V .
$$

The quantity $\sigma[f]$ is related to the orientational entropy,

$$
\sigma[f]=-\frac{S_{\text {or }}}{N k}=\int f(\Omega) \ln [4 \pi f(\Omega)] \mathrm{d} \Omega .
$$

The contribution $c \rho[f]$ is also entropic in nature namely the second virial contribution to the excluded volume,

$$
\rho[f]=\frac{4}{\pi} \iint\left|\sin \gamma\left(\Omega, \Omega^{\prime}\right)\right| f(\Omega) f\left(\Omega^{\prime}\right) \mathrm{d} \Omega \mathrm{d} \Omega^{\prime},
$$

where $\gamma\left(\Omega, \Omega^{\prime}\right)$ is the angle between two rods with orientations $\Omega$ and $\Omega^{\prime}$.

The isotropic-nematic transition originates from a competition between these two types of entropy: for low concentrations the orientational entropy dominates and is maximized by an isotropic distribution, whereas for high concentrations the excluded volume term becomes more important which favours a nematic distribution. In the isotropic phase all orientations are equally probable which implies in view of the normalization condition (2):

This immediately leads to

$$
f_{\text {iso }}(\Omega)=1 / 4 \pi
$$

$$
\sigma_{\text {iso }}=0, \quad \rho_{\text {iso }}=1,
$$

and therefore the free energy in the isotropic phase takes the form,

$$
\Delta F^{\text {iso }} / N k_{\mathrm{B}} T=\text { const. }+\ln c+c .
$$

To treat the nematic phase we must first minimize the free energy with respect to the orientation distribution function $f$. This can be done formally considering $\Delta F$ as a functional of $f$ while taking into account the normalization condition (2) by adding $\lambda^{\prime} \int f(\Omega) \mathrm{d} \Omega\left(\lambda^{\prime}\right.$ being a Lagrange undetermined multiplier) and then minimizing the resulting expression. This means the functional derivative must be 0 or

$$
\frac{\delta}{\delta f}\left(\frac{\Delta F[f]}{N k T}\right)=\lambda^{\prime}
$$

An alternative route is to choose a (normalized) trial function with one or more variational parameters and then minimize the free energy with respect to these parameters. For illustrative purposes we shall use a gaussian distribution function (Odijk 1986) here

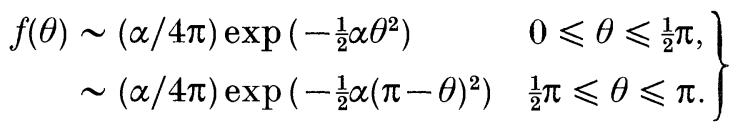


Using the gaussian distribution one obtains to leading order (large $\alpha$ )

$$
\begin{gathered}
\sigma(\alpha) \sim \ln \alpha-1, \\
\rho(\alpha) \sim 4 / \sqrt{ }(\pi \alpha) .
\end{gathered}
$$

Substituting these results in equation (1) yields the following expression for $\Delta F$ valid for large $\alpha$ :

$$
\Delta F(\alpha) / N k T \sim \text { const. }+\ln \alpha-1+4 c / \sqrt{ }(\pi \alpha) .
$$

Minimizing this expression with respect to $\alpha$ gives

$$
\alpha \sim 4 c^{2} / \pi \text {. }
$$

and thus

$$
\Delta F^{\text {nem }} / N k_{\mathrm{B}} T \sim \text { const. }+3 \ln c+\ln (4 / \pi)+1 .
$$

The treatment given above is only valid for $\alpha$ sufficiently large, say $\alpha \geqslant 10$. Comparing the free energies of the isotropic and nematic phase we note that $\Delta F^{\text {nem }}$ becomes smaller than $\Delta F^{\text {iso }}$ for $c>4.03$ which indicates the possibility of a phase transition. To be in mechanical and chemical equilibrium the isotropic and nematic phase must have the same osmotic pressure and the same chemical potential

$$
\Pi^{\text {iso }}\left(c_{i}\right)=\Pi^{\text {nem }}\left(c_{n}\right), \quad \mu^{\text {iso }}\left(c_{i}\right)=\mu^{\text {nem }}\left(c_{n}\right) .
$$

Using the well-known thermodynamic relations

$$
\Pi=-(\partial \Delta F / \partial V)_{N, T, \mu_{0}}, \quad \mu=(\partial \Delta F / \partial N)_{V, T, \mu_{0}},
$$

where $\mu_{0}$ is the chemical potential of the solvent, one obtains (using the free energy expressions (8) and (13) for the isotropic and nematic phase) the following coexistence conditions :

$$
c_{i}\left(1+c_{i}\right)=3 c_{n}, \quad \ln c_{i}+2 c_{i}=3 \ln c_{n}+\ln (4 / \pi)+3 .
$$

From this we find the following coexisting concentrations (Odijk 1986):

$$
c_{i}=3.45, \quad c_{n}=5.12,
$$

implying (via equation (14)),

$$
\alpha=33.4 \text {, }
$$

which is reasonably large (thus justifying the use of the gaussian trial function). Finally, the usual measure of the ordering in the nematic phase is the nematic order parameter given by

$$
S=\left\langle P_{2}(\cos \theta)\right\rangle=\left\langle\frac{3}{2} \cos ^{2} \theta-\frac{1}{2}\right\rangle \sim\left\langle 1-\frac{3}{2} \theta^{2}\right\rangle \sim 1-3 / \alpha=0.910 .
$$

Here we have used that for a gaussian distribution function asymptotically

$$
\left\langle\theta^{2}\right\rangle \sim 2 / \alpha .
$$

The accuracy of the results obtained here with the gaussian distribution function can be assessed by comparing them with the values obtained by an accurate numerical calculation (Lekkerkerker et al. 1984)

$$
c_{i}=3.290, \quad c_{n}=4.191, \quad S=0.7922 .
$$

Onsager's (second virial) theory of the isotropic-nematic transition is accurate for $L / D \gg 1$ (and in fact it is an exact theory for $L / D \rightarrow \infty$ ). The question then arises, 'how long should the rods be for the theory to have quantitative validity?' To quantify the necessary length Straley (1973) considered the first correction term (the 
third virial contribution) to the Onsager theory. Straley estimates that up to $L / D \approx 20$ the contribution of the third virial term to the free energy (evaluated at the transition density) is at least comparable to the contribution of the second virial term. For $L / D$ values as high as 100 he finds that the contribution of the third virial term is still about $10 \%$ of the second term. Using simulation results for the higher virial coefficients of hard spherocylinders (Frenkel 1987a) it turns out that Straley's estimate is remarkably accurate for $L / D=100$ but that for the lower $L / D$ values he is slightly too pessimistic about the quantitative accuracy of the Onsager theory. For example for $L / D=10$ the contribution of the third virial term is certainly not more than $50 \%$ of that of the second virial term. Furthermore simulation results indicate that - whereas the contribution of $B_{3}$ remains substantial up to $L / D \approx 10^{2}$ - the contributions of the higher virial terms decrease very quickly with increasing elongation. This means that for intermediate elongations the incorporation of the third virial contribution in the Onsager theory of the isotropic-nematic phase transition suffices to make the theory quantitatively accurate. Recent calulations by Tjipto-Margo \& Evans (1990) show that this is indeed the case for length-to-breadth ratios of 10 while even for length-to-breadth ratios as small as 5 incorporation of the third virial contribution leads to results that lie only about $10 \%$ above Monte Carlo results.

For elongations below five the second and third virial terms do not suffice to make the theory for the isotropic-nematic phase transition quantitatively accurate. In fact it may be surmized that all higher virial coefficients have to be somehow incorporated in the theory. Unfortunately at the present time the statistical mechanics of fluids of rod-like particles is not sufficiently well developed to provide confident answers on how to do this. Nevertheless a number of interesting attempts have been made to incorporate the higher virial coefficients, which we recently reviewed (Vroege \& Lekkerkerker 1992).

\section{(b) Transitions to smectic and crystal phases}

Onsager (1949) already mentioned the possibility that in dispersions of thin hard rods other types of anisotropic phases such as crystals and smectic liquid crystals might occur. This suggestion did not receive much attention for a long time. Indeed the statistical mechanical calculations for phase transitions involving these phases, which exhibit periodic variations in the density in one (smectic) or three (crystal) dimensions, are much more difficult than those for the isotropic-nematic phase transition. This is a situation where computer simulations turned out to be of great assistance. Over the last 10 years Frenkel and coworkers (Frenkel et al. 1984, 1988; Frenkel \& Mulder 1985; Frenkel 1987a,b, 1988, 1989; Allen et al. 1989; Veerman \& Frenkel 1990) have reported extensively on simulations of phase behaviour for both hard ellipsoids of revolution and hard spherocylinders. For further details see Allen (this volume), where computer simulations of hard core models are reviewed.

\section{Liquid crystal phase transitions in polydisperse dispersions of thin hard rods}

(a) The isotropic-nematic transition

In an experimental situation rigid colloidal particles will hardly ever be truely monodisperse. This may have rather strong effects on the isotropic-nematic phase transition. Onsager (1949) already hinted at this in his original paper and even 
devoted an appendix to calculating the required integrals in bidisperse systems for his choice of the orientational distribution function. This problem was attacked by one of us and coworkers (Lekkerkerker et al. 1984) with numerical calculations based on series expansions of the orientational distribution function. Several interesting results like a strong fractionation effect (with the longer rods going preferentially to the nematic phase) and a widening of the biphasic gap emerged from these calculations. A few years ago Birshtein et al. (1988), using the same method, also located a triphasic (one isotropic and two nematic phases) and nematic-nematic area in a bidisperse system with length ratio 5, which were not detected by Lekkerkerker et al. (1984).

The above mentioned calculations are all based upon numerical procedures and consequently tend to obscure the physical mechanism involved. For this reason an analytical theory may be helpful for a better understanding. Like in the monodisperse case the gaussian distribution function allows one to make such analytical calculations for the bidisperse case (Odijk \& Lekkerkerker 1985). Extending Onsager's treatment for the monodisperse case to bidisperse systems, the free energy of $N_{1}$ rigid rods with length $L_{1}$ and diameter $D$ and $N_{2}$ rigid rods with the same diameter and a (larger) length $L_{2}$ in a dispersion with volume $V$ can be written as

$$
\begin{aligned}
\Delta F\left[f_{1}, f_{2}\right] /\left(N_{1}+N_{2}\right) k_{\mathrm{B}} T=\text { const. }+\ln c+(1-x) \ln (1-x) \\
\quad+x \ln x+(1-x) \sigma_{1}+x \sigma_{2}+c\left[(1-x)^{2} \rho_{11}+2 x(1-x) q \rho_{12}+x^{2} q^{2} \rho_{22}\right] .
\end{aligned}
$$

Here $q=L_{2} / L_{1}$ is the length ratio of the longer and the shorter rods, $x=N_{2} /\left(N_{1}+N_{2}\right)$ is the mole fraction of the longer rods and $c$ is the dimensionless concentration defined as

$$
c=\frac{1}{4} \pi L_{1}^{2} D\left(N_{1}+N_{2}\right) / V .
$$

The quantities $\sigma_{j}$ and $\rho_{j k}$ are the appropriate generalizations of the corresponding terms for the monodisperse case (equations (4) and (5)). In the isotropic phase they have again the values,

$$
\sigma_{j}=0, \quad \rho_{j k}=1, \quad j, k=1,2,
$$

and thus the free energy takes the form

$$
\Delta F^{\text {iso }} /\left(N_{1}+N_{2}\right) k_{\mathrm{B}} T=\text { const. }+\ln c+(1-x) \ln (1-x)+x \ln x+c((1-x)+q x)^{2} .
$$

Using in the nematic phase for the orientation distribution functions $f_{1}$ and $f_{2}$ gaussians with parameters $\alpha_{1}$ and $\alpha_{2}$ one now obtains the following expressions

$$
\begin{gathered}
\sigma_{j} \sim \ln \alpha_{j}-1, \\
\rho_{j k} \sim 4\left(\alpha_{j}+\alpha_{k}\right)^{\frac{1}{2}} /(2 \pi)^{\frac{1}{2}} \alpha_{j}^{\frac{1}{2}} \alpha_{k}^{\frac{1}{k}} .
\end{gathered}
$$

Using these expressions in equation (24) and minimizing with respect to $\alpha$, gives us

$$
\frac{1}{2} \pi^{\frac{1}{2}} c^{-1} \alpha_{1}^{\frac{1}{1}}=(1-x)+2^{\frac{1}{2}} x q h(Q)
$$

with definitions

$$
\begin{gathered}
Q \equiv \alpha_{2} / \alpha_{1}, \\
h(Q) \equiv Q^{\frac{1}{2}} g(Q) \equiv(Q /(Q+1))^{\frac{1}{2}} .
\end{gathered}
$$

A similar equation as (30) is obtained by minimizing the free energy with respect to $\alpha_{2}$; both equations may be combined to obtain an expression only involving the ratio of both $\alpha \mathrm{s}$

$$
Q^{\frac{1}{2}}=q\left[x q+2^{\frac{1}{2}} g(Q)(1-x)\right] /\left[2^{\frac{1}{2}} h(Q) x q+(1-x)\right] .
$$



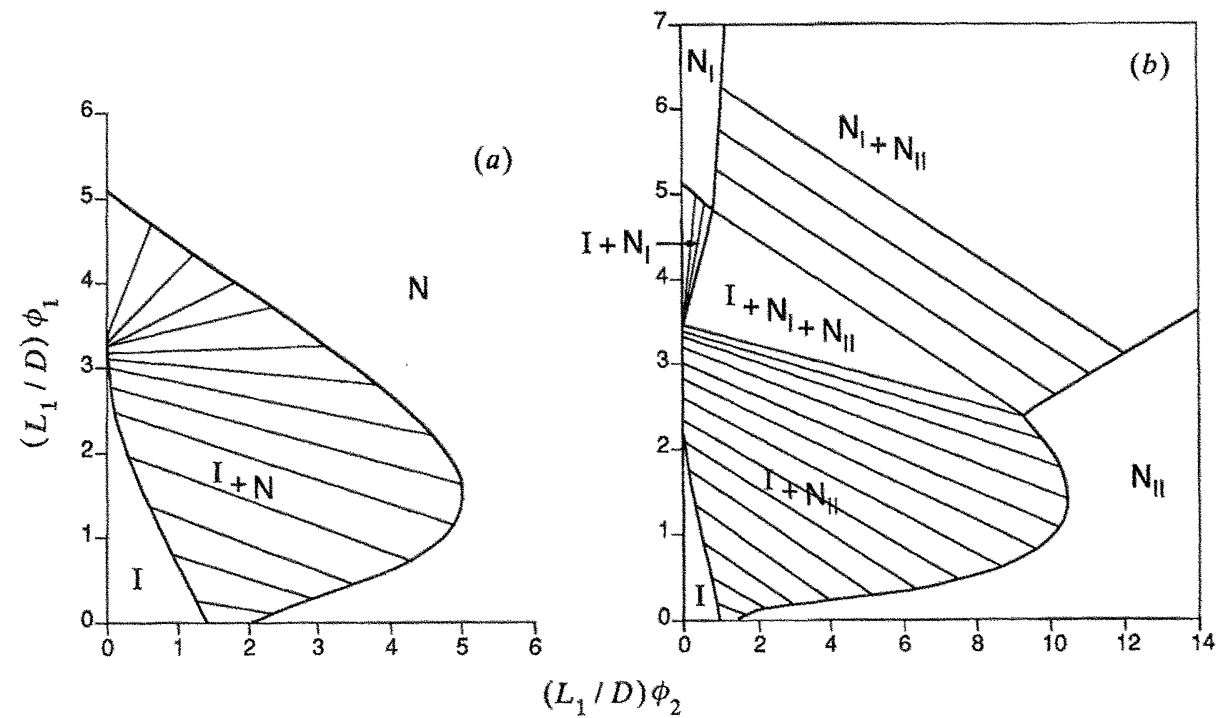

Figure 1. Phase diagrams for a bidisperse system of rigid hard rods in terms of volume fractions $\phi_{1}$ and $\phi_{2}$ with length ratios $(a) q=2.5,(b) q=3.5$. To avoid the need to fix aspect ratio $L_{1} / D$, both volume fractions were multiplied by this number. Thick lines indicate phase boundaries, thin lines represent tie lines connecting coexisting phases.

From this equation it follows that $Q$ only depends on the mole fraction $x$ and not on the concentration $c$. This implies that the concentration dependence of the $\alpha \mathrm{s}$ remains quadratic like in the monodisperse case, which in turn leads to an excluded volume term in the free energy which does not depend on $c$ (like in the monodisperse case). In fact this term turns out to be simply $2\left(N_{1}+N_{2}\right) k T$ and the free energy takes the form

$$
\begin{aligned}
\Delta F^{\mathrm{nem}} /\left(N_{1}+N_{2}\right) k_{\mathrm{B}} T=\text { const. } & +3 \ln c+\ln (4 / \pi)+1+x \ln x+(1-x) \ln (1-x) \\
& +2 \ln \left[(1-x)+2^{\frac{1}{2}} x q h(Q)\right]+x \ln Q .
\end{aligned}
$$

To locate phase transitions we must solve the coexistence conditions equating the osmotic pressure and chemical potentials in the coexisting phases. These can be obtained from the free energy expressions (27) and (34). This leads to surprisingly simple coexistence relations which give rise to different phase behaviour depending on the length ratio (Vroege \& Lekkerkerker 1993).

(i) $1<q<3.1672$

For low concentrations the isotropic phase is stable whereas for high concentrations the nematic phase is stable. These two monophasic regions are separated by a biphasic isotropic-nematic region. This biphasic region is wider than in the monodisperse case (the more so with increasing $q$ ). In addition there is a strong fractionation effect with the longer rods collecting preferentially in the anisotropic phase. All these features appear in figure $1 a$ where the phase diagram for $q=2.5 \mathrm{in}$ terms of volume fractions $\phi_{1}$ and $\phi_{2}$ is represented.

(ii) $q>3.1672$

Now a three-phase region in which an isotropic phase coexists with two nematic phases as well as three biphasic regions (two isotropic-nematic coexistence regions and a region where two nematic phases coexist) appear in the phase diagram. This 
is illustrated in figure $1 b$ where the phase diagram for $q=3.5$ is represented. Remarkably the underlying mechanism for the nematic-nematic phase transition does not involve changes in excluded volume but a balance between orientational entropy and entropy of mixing (Vroege \& Lekkerkerker 1993).

\section{(b) Liquid crystalline phases with partial translational order}

Very little is known about the influence of length polydispersity on the formation of liquid crystalline phases with partial translational order. Sluckin (1989) extended the work of Mulder (1987) on the nematic-smectic transition in a monodisperse system of parallel hard rods to a polydisperse system of such rods. He showed that the smectic transition is postponed to higher densities and estimated that for $\left.\left\langle(L-\langle L\rangle)^{2}\right\rangle /\langle L\rangle^{2}\right\rangle 0.09$ all smecticity will be destroyed. Recently Stroobants (1992) presented Monte Carlo simulations of binary mixtures of hard parallel spherocylinders with length-to-width ratios $L_{1} / D=1.0$ and $L_{2} / D$ ranging from 1.3 to 2.1. For $L_{2} / D>1.6$ a thermodynamically stable columnar phase is observed which is not formed by the monodisperse component separately. The nematic-smectic transition, which is postponed by increasing the $L_{2} / D$ ratio, is eventually preempted by a nematic-columnar transition for $L_{2} / D>1.9$ showing that in these mixtures bidispersity favours columnar order over smectic order.

\section{Charged rods}

In the preceding sections we only considered hard core interactions, to which we will now add electrostatic repulsion. When the rods are charged there is an additional soft repulsion because of the interaction between the electric double layers of the polyelectrolytes. Onsager (1949) already indicated that the effect of the electrostatic interaction will be equivalent to an increase of the effective diameter. Many years later Stroobants et al. (1986) noted that this is not the only effect of the electrostatic interaction. The electrostatic repulsion depends on orientation and thus the effect of the electrostatic repulsion will be different in the isotropic phase from that in the nematic phase. In the remainder of this section we closely follow the treatment for charged rods given in Stroobants et al. (1986).

For the evaluation of the free energy we need to know the potential of mean force $w$ of this interaction. Although the potential of mean force for two charged rods is, in general, very difficult to evaluate, we are helped by the fact that we only need to know the Mayer function $\Phi=\exp (-w / k T)-1$. The Mayer function is insensitive to the exact form of the potential when $w / k T \gg 1$ in which case $\Phi \approx-1$ and its contribution to the second virial term in the free energy is always the same. This implies that we only need to know an accurate form of the potential of mean force for configurations where the outer parts of the double layers overlap. In that case we can approximate $w$ by the interaction between two effective line charges in the Debye-Hückel approximation which is well known (Brenner \& Parsegian 1974; Stigter 1977; Fixman \& Skolnick 1978)

$$
w^{\mathrm{e} 1} / k T=A \mathrm{e}^{-\kappa x} / \sin \gamma
$$

with

$$
A=2 \pi \nu_{\text {eff }}^{2} Q \kappa^{-1} \text {. }
$$

Here $x$ is the shortest distance between the two lines, $\gamma$ is their mutual angle, $\nu_{\text {eff }}$ is the (effective) linear charge density (i.e. number of charges per unit length), $\kappa^{-1}$ is the 


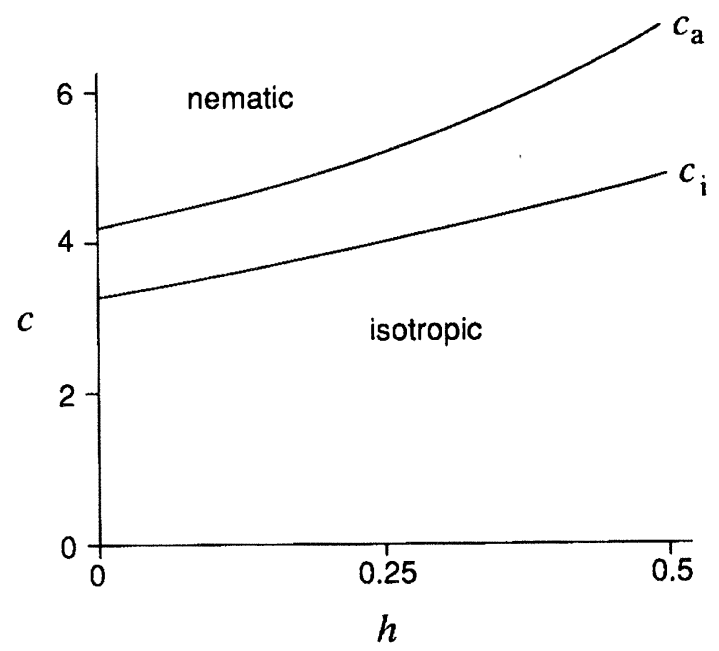

Figure 2. The effect of electrostatic twisting on the isotropic-nematic phase transition. Note that we plot effective concentration $c$ against $h$.

Debye screening length and $Q$ is the Bjerrum length $e^{2} / 4 \pi \epsilon_{0} \epsilon k T$ with $\epsilon$ the dielectric permittivity. For details on how to relate $\nu_{\text {eff }}$ to the relevant characteristics of the polyelectrolyte (surface charge density, diameter, ionic strength) we refer to the work of Stroobants et al. (1986). With expression (35) it is possible to evaluate the free energy in the second virial approximation leading to

$$
\Delta F[f] / N k T=\text { const. }+\ln c+\sigma[f]+c(\rho[f]+h \eta[f]) .
$$

Here $\sigma[f]$ and $\rho[f]$ have the usual meaning. The dimensionless concentration $c$ is now defined as in equation (3) but with the diameter replaced by the effective diameter

$$
D_{\text {eff }}=D+\kappa^{-1}\left(\ln A^{\prime}+c_{E}+\ln 2-\frac{1}{2}\right),
$$

where $c_{E}=0.577215$ is Euler's constant and $A^{\prime}=A \mathrm{e}^{-\kappa D}$. The second term on the right-hand side of (38) represents the increase of the effective diameter due to the electrostatic repulsion between the rods. In addition to the larger effective diameter the electrostatic interaction shows up in the extra term

$$
\eta[f] \equiv \frac{4}{\pi} \iint\left[-\sin \gamma \ln (\sin \gamma)-\left(\ln 2-\frac{1}{2}\right) \sin \gamma\right] f(\Omega) f\left(\Omega^{\prime}\right) \mathrm{d} \Omega \mathrm{d} \Omega^{\prime},
$$

which is called the twisting effect as it originates from the factor $(\sin \gamma)^{-1}$ in equation (35). The relative importance of this effect is determined by the parameter

$$
h \equiv\left(\kappa D_{\text {eff }}\right)^{-1} \text {. }
$$

Stroobants et al. (1986) determined the isotropic-nematic phase transition from the free energy (37). As the scaled concentration now is based on the larger effective diameter the phase transition is shifted to lower particle number densities. On the other hand the nematic phase is destabilized by the twisting effect: charged rods want to be perpendicular. This second effect of charge is seen in figure 2. However, in general the stabilizing effect on the nematic state of a larger effective diameter dominates the destabilizing twisting effect.

Further developments of the effect of electrostatic interactions on the isotropic-nematic phase transitions in dispersions of rigid rodlike polyelectrolytes have 
been given by several authors. Sato \& Teramoto (1991) (also see Sato et al. 1990) incorporated end effects of the electrostatic interaction and took into account that the different concentrations of polyelectrolyte rods in the coexisting isotropic and nematic phase will lead to different salt concentrations in these phases (Donnan effect) and thus to different Debye screening lengths. Nyrkova \& Khokhlov (1986) presented theoretical evidence for the occurrence of two nematic phases of different degrees of order under certain special conditions. Deutsch \& Goldenfeld $(1982 a, b)$ have given a treatment for the case where the hard core diameter is zero and the isotropic-nematic transition is solely caused by the long-range electrostatic interactions between infinitely thin needles.

\section{Attractive interactions}

To treat the effect of attractive interactions on the isotropic-nematic phase transition satisfactorily is rather more delicate than the incorporation of electrostatic repulsion. This stems from the fact that in a solution of rodlike particles the translational degrees of freedom are strongly coupled to their orientational degrees of freedom. Since parallel configurations show the largest attraction these configurations dominate the problem whenever two rods approach each other. The orientational bias exerted by repulsive interactions (hard core, electrostatic) is a great deal weaker.

So far the effect of attractions has been incorporated on the level of a van der Waals mean field theory (Gelbart \& Barron 1977; Cotter 1977; Khokhlov \& Semenov 1985). In these theories the effect of attractions is included in the free energy by means of a concentration dependent mean field potential of the form

$$
U_{\text {att }}=-u_{0} c-u_{\mathrm{a}} c\left\langle P_{2}(\cos \theta)\right\rangle P_{2}(\cos \theta)
$$

with $u_{0}$ and $u_{\mathrm{a}}$ unspecified constants. Whereas the work of Gelbart \& Barron (1977) and Cotter (1977) was directed towards thermotropic liquid crystals, Khokhlov \& Semenov (1985) specifically considered the effect of attractive interactions in dispersions of rodlike particles. For the hard core part of the free energy one must now include higher virial terms since in a situation with many nearly parallel configurations the second virial term no longer exceeds the higher virial terms. Khokhlov \& Semenov do this by using the Parsons approach (Parsons 1979) which may be considered an extension of the semi-empirical Carnahan-Starling equation for hard spheres to the case of rodlike particles. They find three different scenarios for the phase behaviour which are schematically illustrated in figure 3 . For large $L / D$ ratios they predict that the attractive interaction leads to two nematic phases with different concentrations and order parameters and for low $L / D$ ratios the attractive interaction leads to two isotropic phases (a 'dilute' phase and a 'concentrated' phase, the equivalent of the gas and the liquid phase in an atomic system). For intermediate $L / D$ ratios there is just an isotropic and a nematic phase. The possibility that attractive interactions may give rise to two nematic phases was already predicted long ago by Flory (1956), using a lattice theory.

Given the subtle nature of the effect of attractive interactions in a solution of rodlike particles one may wonder whether a treatment on the level of the van der Waals mean field approximation can be at all satisfactory. Van der Schoot \& Odijk (1992) have shown that as soon as attractive interactions mildly influence the second virial contribution the effect on the third virial coefficient is considerable. This casts doubt 

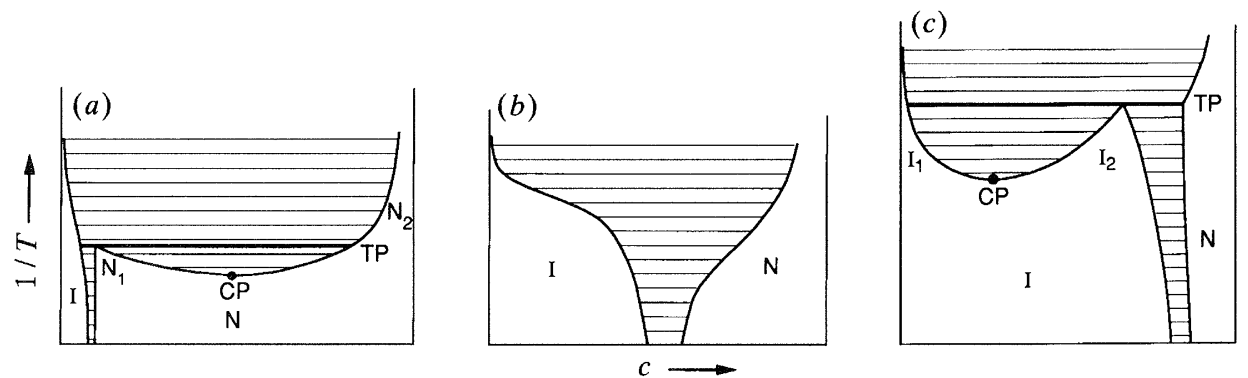

Figure 3. Schematic phase diagrams for dispersions of rigid rods with attractive interactions showing concentration of the rods against inverse temperature for $(a) L / D \approx 50,(b) L / D \approx 5,(c)$ $L / D \approx 2$. Tie lines are shown in the two-phase regions. $I, I_{1}$ and $I_{2}$ refer to isotropic fluid phases and $\mathrm{N}, \mathrm{N}_{1}, \mathrm{~N}_{2}$ to nematic liquid crystal phases. In $(a)$ and $(c)$ the critical point $(\mathrm{CP})$ and triple point (bold line marked TP) are also indicated. (After Khokhlov \& Semenov 1985.)

on the use of virial expansions for this case and may be at the origin of the strong tendency towards gelation in solutions of rodlike particles on lowering the temperature.

\section{Liquid crystal phase transitions in solutions of semi-flexible molecules}

\section{(a) Introduction}

As indicated in the preceding the Onsager theory is at the least conceptually very important for understanding lyotropic liquid crystals. Besides extensions with effects of polydispersity, charge, etc., the incorporation of the influence of (slight) fluctuations of the molecules has appeared to be essential to explain experiments with stiff polymers. On transition to an orientationally ordered phase, chain molecules not only lose orientational entropy but the number of accessible conformations is constricted at the same time. It took a long time since Onsager before this effect was taken into due account, starting around 10 years ago (Khokhlov \& Semenov 1981).

Before discussing the various methods to describe the loss of entropy of confined chains we shall first briefly sketch the wormlike chain model of a polymer (Kratky \& Porod 1949; Yamakawa 1971, 1984). This theoretical model is much used for polymers which gradually change their direction through small fluctuations in bond lengths and angles. The polymer is considered to be a continuous elastic line which is described by its direction (unit) vector $\hat{\boldsymbol{u}}(s)$ at each point $s$ (measured along its contour). A measure for the chain stiffness is its persistence length $P$ which represents the length scale on which a chain loses its original direction

$$
\langle\hat{\boldsymbol{u}}(s) \cdot \hat{\boldsymbol{u}}(s+\Delta s)\rangle=\exp (-|\Delta s| / P),
$$

where $\langle\ldots\rangle$ indicates an average over all chain conformations. The conformational energy of a free wormlike chain may be formulated in terms of its bending elastic energy

$$
U_{\text {bend }}=\frac{1}{2} \epsilon \int_{0}^{L}\left(\frac{\partial \hat{\boldsymbol{u}}(s)}{\partial s}\right)^{2} \mathrm{~d} s=\frac{1}{2} \epsilon \int_{0}^{L}\left(\frac{\partial^{2} \boldsymbol{r}(s)}{\partial s^{2}}\right)^{2} \mathrm{~d} s,
$$

where bending elastic constant $\epsilon$ is related to the persistence length

$$
\epsilon \equiv P k_{\mathrm{B}} T \text {. }
$$

Phil. Trans. R. Soc. Lond. A (1993) 


\section{(b) Conformational free energy: Khokhlov-Semenov theory}

The method applied by Khokhlov \& Semenov $(1981,1982)$ leads to an expression for the conformational free energy as a functional of an orientational distribution function $f(\theta)$ which here represents the distribution of angles $\theta$ between the direction $\hat{\boldsymbol{u}}$ of a segment and the average direction given by director $\hat{\boldsymbol{n}}$. We will only consider the simplest version of this theory for very long chains $(L \gg P$; also see Odijk 1986; Vroege \& Lekkerkerker 1992) where the distribution function does not depend on the position of the segment on the chain. Khokhlov \& Semenov (1981) extended a theory by Lifshitz (Lifshitz 1968; Lifshitz et al. 1978) for the entropy of a spatially confined gaussian chain to the case of an orientationally confined wormlike chain.

They start from the (unnormalized) configurational partition function $Z\left(\hat{\boldsymbol{u}}^{\prime}, \hat{\boldsymbol{u}} ; 0, L\right)$ for a wormlike chain with fixed end directions $\hat{\boldsymbol{u}}(0)=\hat{\boldsymbol{u}}^{\prime}$ and $\hat{\boldsymbol{u}}(L)=\hat{\boldsymbol{u}}$, placed in an external potential $U_{\text {ex }}(\hat{\boldsymbol{u}})$. This function $Z$ may be shown to fulfil

$$
\partial Z / \partial L=(1 / 2 P) \Delta_{\hat{u}} Z-\left(U_{\mathrm{ex}}(\hat{\boldsymbol{u}}) / k T\right) Z,
$$

which is a kind of diffusion equation with contour length $L$ playing the role of time. Here direction vector $\hat{\boldsymbol{u}}$ diffuses over a unit sphere $\left(\Delta_{\hat{u}}\right.$ is the two-dimensional laplacian on the unit sphere) with diffusion constant $(2 P)^{-1}$ in the externally applied potential $U_{\text {ex }}(\hat{\boldsymbol{u}})$. If $Z$ is developed in an eigenfunction expansion it will be dominated by the largest eigenvalue $\lambda_{0}$ and its eigenfunction $\phi_{0}$

$$
Z\left(\hat{\boldsymbol{u}}^{\prime}, \hat{\boldsymbol{u}} ; 0, L\right) \sim \mathrm{e}^{\lambda_{0} L} \phi_{0}\left(\hat{\boldsymbol{u}}^{\prime}\right) \phi_{0}(\hat{\boldsymbol{u}}) .
$$

In this case orientational distribution function $f(\hat{\boldsymbol{u}})(=f(\Omega))$ is simply given by

$$
f(\hat{\boldsymbol{u}}) \sim \phi_{0}^{2}(\hat{\boldsymbol{u}}) .
$$

By subtracting from (the extensive part of) the free energy, derived from (44),

$$
F=-k T \ln \langle\langle Z\rangle\rangle_{\hat{u}^{\prime}, \hat{u}} \sim-k T \lambda_{0} L
$$

the internal energy $U$ resulting from the externally applied field

$$
U=L \int U_{\text {ex }}(\hat{\boldsymbol{u}}) f(\hat{\boldsymbol{u}}) \mathrm{d} \hat{\boldsymbol{u}}
$$

the conformational free energy is now simply obtained

$$
F_{\text {con }}=F-U=-k T L \int\left[\lambda_{0}+\frac{U_{\text {ex }}(\hat{\boldsymbol{u}})}{k T}\right] \phi_{0}^{2}(\hat{\boldsymbol{u}}) \mathrm{d} \hat{\boldsymbol{u}} \equiv-T S_{\text {con }} .
$$

Here we have used the normalization of $f(\hat{\boldsymbol{u}})$. The force of this method originates from the fact that the expression in square brackets in (48) is also obtained by substituting (44) in (43) and may be eliminated,

$$
S_{\text {con }}=\frac{1}{2} k \frac{L}{P} \int \phi_{0}(\hat{\boldsymbol{u}}) \Delta_{\hat{\boldsymbol{u}}} \phi_{0}(\hat{\boldsymbol{u}}) \mathrm{d} \hat{\boldsymbol{u}}=\frac{1}{2} k \frac{L}{P} \int f^{\frac{1}{2}}(\Omega) \Delta f^{\frac{1}{2}}(\Omega) \mathrm{d} \Omega,
$$

where the notation of the second part of this equation is more in line with the rest of this paper. We have now obtained an expression for $S_{\text {con }}$-independent of the external field $U_{\text {ex }}(\hat{\boldsymbol{u}})$ - in terms of the angular distribution function $f(\Omega)$, analogous to (4) for rods. If we add an interaction term to the free energy we can now proceed Phil. Trans. R. Soc. Lond. A (1993) 
similarly to the Onsager theory for rods. For illustrative purposes we may use a gaussian distribution function (10) in (49) to obtain

$$
S_{\text {con }}(\alpha) \sim-\frac{1}{4} k(L / P) \alpha .
$$

\section{(c) The isotropic-nematic phase transition}

To find the phase transition we need an interaction term besides the conformational entropy. For rods the second virial term in the free energy scales like $N^{2} L^{2}$ (cf. equations (1) and (3)), which implies it is invariant to subdividing the rods into smaller rods while keeping the total length $N L$ in the system constant. This implies that there is no need for a long-range correlation between segments as long as the local interaction is rodlike in character. Therefore, to first order the interaction term for very stiff wormlike chains is the same as the second virial term for rods

$$
F_{\text {int }} / N k T=c \rho[f] \text {. }
$$

For the nematic the total free energy is obtained by combining (50), (51) and (12),

$$
\Delta F^{\text {nem }} / N k T \sim \text { const. }+(L / P)\left[\frac{1}{4} \alpha+c_{P} 4 / \sqrt{ }(\pi \alpha)\right],
$$

where we introduced a new dimensionless concentration

$$
c_{P} \equiv(P / L) c .
$$

We may neglect the translational entropy term $\ln c$, since it is $O(P / L)$ smaller than the other terms of (52) for the pertinent value of $c_{P}$ (see (55) and (56)). Minimizing expression (52) with respect to $\alpha$ gives

$$
\alpha \sim 4 c_{P}^{\frac{2}{3}} / \pi^{\frac{1}{3}}
$$

Note that the concentration dependence is much weaker than for rods (14). A similar neglect of the logarithmic term in (8) yields

$$
\Delta F^{\text {iso }} / N k T \approx \text { const. }+(L / P) c_{P} .
$$

Now, solving coexistence equations (16) and (17) leads to the coexisting concentrations (Odijk 1985)

$$
c_{P, i}=7.77, \quad c_{P, n}=9.71,
$$

with $\alpha=12.43$. This low value of $\alpha$ only provides qualitative significance to this result. More precise values are obtained by numerical calculations (Vroege \& Odijk 1988)

$$
c_{P, i}=5.124, \quad c_{P, n}=5.509 .
$$

Because of definition $\left(52^{\prime}\right)$ the transition takes place at a roughly $L / P$ times higher volume fraction than for rods of the same length. Although it is tempting to view the chain as built up from $L / P$ effectively rod-like segments, this is not correct. This is already clear from the non-logarithmic dependence of $S_{\text {con }}$ in (50). In the next section we describe a physically more realistic picture of the nematic state.

\section{(d) The Odijk deflection length}

In 1983 Odijk (see also Odijk 1986, 1988) introduced a scaling approach to the problem of a confined wormlike chain. When we follow a wormlike chain along its contour it will behave like a free chain as long as there is no external influence either from a neighbouring chain or from a wall. In this stage expressions like (41) for a free wormlike chain still apply. At larger length scale, however, the chain will experience 


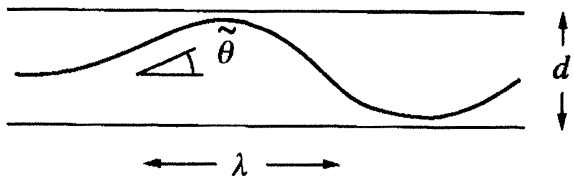

Figure 4. A chain in a tube of diameter $d$ is confined by the walls to remain within an angle of about $\tilde{\theta}$ from the axis of the tube. Therefore the typical length scale of fluctuations is given by deflection length $\lambda$ (see text).

the restriction either because it is forced to remain within a certain angle with the director or because it cannot penetrate the tube wall. The deflection length $\lambda$ marks the transition from the free to the constricted régime. It is derived from (41) which we may Taylor expand for the small angles and distances involved, whence:

$$
\left\langle\theta^{2}\right\rangle \approx 2|\Delta s| / P \quad \theta \ll 1,|\Delta s| \ll P .
$$

For a nematically constricted chain a typical value for $\left\langle\theta^{2}\right\rangle$ is given by (22). Therefore, the length scale $\lambda$ follows from equating both expressions

$$
\lambda \sim P / \alpha \quad \text { (nematic confinement). }
$$

If we look at a chain confined to a tube we must take the spatial constriction into consideration. A similar expression to (58) may be derived from the deviation in the lateral direction. The deflection length is the length scale on which this deviation equals the tube radius. In figure 4 we sketch a connection between the spatial deviation and the angular deviation at $|\Delta s|=\lambda$

$$
\left\langle\theta^{2}\right\rangle^{\frac{1}{2}} \sim d / \lambda \text {. }
$$

Substituting this expression into (57) leads to an expression for $\lambda$ in a tube

$$
\lambda \sim d^{\frac{2}{3}} P^{\frac{1}{3}} \quad \text { (spatial confinement). }
$$

An important conclusion from both (58) and (60) is that the deflection length $\lambda$ is much smaller than persistence length $P$ for strongly confined chains. At the deflection points the chain will be obstructed to take a free conformation and will be deflected towards a more restricted conformation. At the same time the chain will lose the correlation with its original direction or position. Therefore, the correlation length for a free chain $P$ is replaced by a much smaller correlation length $\lambda$.

A scaling analysis for a very long chain gives the confinement free energy by requiring the free energy to be extensive, i.e. proportional to the total length $L$ which must be divided by the only relevant length scale left in the problem:

$$
F_{\text {con }} \sim k T L / \lambda \text {. }
$$

Combining this with the expression (58) for $\lambda$ in the nematic state leads to an expression

$$
S_{\text {con }} \equiv-F_{\text {con }} / T \sim-k(L / P) \propto,
$$

which is similar to (50) apart from the numerical factor which appears to be $\frac{1}{4}$. In the next sections there will be more indications that $\lambda$ is the dominating length scale of the problem of confined chains.

\section{(e) The Helfrich method}

Another approach to confined elastic cylinders is due to Helfrich and Harbich (1985) analogous to Helfrich's theory for the steric repulsion between fluctuating membranes (the undulation force (Helfrich 1978)). This theory may be formulated 
both for spatial and orientational confinement, which we will discuss in parallel. Here we restrict the discussion to strongly confined chains with direction vector $\hat{\boldsymbol{u}}$ almost parallel to $z$ :

$$
\hat{\boldsymbol{u}} \approx\left(\hat{u}_{x}, \hat{u}_{y}, \mathbf{1}\right)
$$

which is the relevant (angular) variable for orientational confinement. For spatial confinement we must use a spatial variable which we define in terms of displacements $a_{x}$ and $a_{y}$ orthogonal to the main (z) direction of the chain,

$$
\boldsymbol{r}=\left(a_{x}, a_{y}, z\right) .
$$

Since $\partial z / \partial s \approx 1$ both quantities are related by the usual relation

$$
\hat{\boldsymbol{u}}=\partial \boldsymbol{r} / \partial \boldsymbol{x} \approx\left(\partial a_{x} / \partial s, \partial a_{y} / \partial s, 1\right)
$$

The deviations in direction or position may be developed in Fourier series

$$
\hat{u}_{i}=\sum_{q} \hat{u}_{q, i} \mathrm{e}^{i q s} \quad \text { and } \quad a_{i}=\sum_{q} a_{q, i} \mathrm{e}^{i q s} .
$$

Substitution in (42) leads to quadratic terms in the free energy for a free chain to which the equipartition theorem may be applied

$$
\frac{1}{2} P k T q^{2}\left\langle\left|\hat{u}_{q, i}\right|^{2}\right\rangle_{\text {free }} L\left(=\frac{1}{2} P k T q^{4}\left\langle\left|a_{q, i}\right|^{2}\right\rangle_{\text {free }} L\right)=\frac{1}{2} k T .
$$

Helfrich's suggestion is that, when the chain is confined, this is causing an increase of the force constant (proportional to $\left\langle\left|a_{i, q}\right|^{2}\right\rangle^{-1}$ ) by the same amount $\tau$ for each Fourier mode

$$
1 /\left\langle\left|a_{q, i}\right|^{2}\right\rangle_{\text {restr }}=1 /\left\langle\left|a_{q, i}\right|^{2}\right\rangle_{\text {free }}+\tau
$$

while Odijk (1986) applied the same recipe to the orientational fluctuations

$$
1 /\left\langle\left|\hat{u}_{q, i}\right|^{2}\right\rangle_{\text {restr }}=1 /\left\langle\left|\hat{u}_{q, i}\right|^{2}\right\rangle_{\text {free }}+\sigma .
$$

To fix $\tau,\left\langle|a|^{2}\right\rangle$ is calculated by summing over the Fourier modes and equated to $\mu d^{2}$ with $d$ the tube diameter and $\mu$ a constant somewhat less than 1 . This gives

$$
\tau / L=1 / 4 \mu^{\frac{4}{3}} d^{\frac{8}{3}} P^{\frac{1}{3}} .
$$

The confinement free energy is obtained from the plausible expression

$$
\Delta F_{\text {con }}=-\frac{1}{2} k T \sum_{q, i} \ln \frac{\left\langle\left|a_{q, i}\right|^{2}\right\rangle_{\text {restr }}}{\left\langle\left|a_{q, i}\right|^{2}\right\rangle_{\text {free }}}=\frac{k T L}{\mu^{\frac{1}{3}} P^{\frac{1}{3}} d^{\frac{2}{3}}} .
$$

This is an expression for the confinement free energy similar to (60) and (61). For orientational confinement this scheme leads to a similar expression, now by relating

in $(22)$. We then find

$$
\sum_{q, i}\left\langle\left|\hat{u}_{q, i}\right|^{2}\right\rangle \text { to }\left\langle\theta^{2}\right\rangle
$$

analogous to $(50)$.

$$
\Delta F_{\text {con }}=\frac{1}{4} k T(L / P) \propto
$$

\section{(f) Computer simulations of a confined wormlike chain}

To check the theories for the confinement free energy of a wormlike chain Dijkstra et al. (1993) simulated a discrete version of this chain in a tube. For the internal potential energy they took the sum of the bending energies of individual joints

$$
U_{\hat{u}_{i}, \hat{u}_{i-1}}=(\epsilon / 2 l)\left(\theta_{\hat{u}_{i}, \hat{u}_{i-1}}\right)^{2},
$$


where $l$ is the segment length (chosen as the unit of length) and $\theta_{\hat{u}_{i}, \hat{u}_{i-1}}$ is the angle between adjacent segments. If summed over all chain segments (73) is a discretization of (42). By devising an appropriate MC sampling scheme they were able to simulate the confinement free energy by an extension of Widom's particle-insertion method. A typical example is $L=512, P=60$ and tube diameter $d=0.60$, all measured in units of segment length. By varying these different parameters the following expression for the free energy was found

$$
\Delta F_{\text {con }} / k_{\mathrm{B}} T=(2.46 \pm 0.07) L / d^{-0.66 \pm 0.07} P^{-0.31 \pm 0.05} .
$$

The linear dependence on $L$ was found for $L>200$. The expression found is in good agreement with (61) and (71), although the prefactor is somewhat different as might be expected.

\section{(g) Extensions}

Recently, Selinger \& Bruinsma (1991) devised a more general approach for infinitely long chains without hairpins, closely related to Helfrich's theory (Helfrich \& Harbich 1985). Inspired by the importance of the Fourier modes in Helfrich's theory, their theory can be viewed as starting from a more general expression for the mean square deviations

$$
\left\langle\left|a_{q}\right|^{2}\right\rangle=2 k T / g(q)
$$

with $\boldsymbol{a}_{q} \equiv\left(a_{q, x}, a_{q, y}\right)$. The distribution for each mode is postulated to be gaussian, hence

$$
\rho\left(\boldsymbol{a}_{q}\right)=(g(q) / 2 \pi k T) \exp \left(-(g(q) / 2 k T)\left|\boldsymbol{a}_{q}\right|^{2}\right)
$$

and a functional integration over all modes is implied. Now an expression for the confinement free energy may be obtained

$$
\frac{F_{\text {con }}}{N k T}=\frac{L}{2 \pi} \int \mathrm{d} q\left[\ln g(q)+\frac{\epsilon q^{4}}{g(q)}\right]
$$

This expression also contains an elastic contribution (from (42)) which represents a decrease in elastic energy when the chain is confined and therefore less bent.

For the interaction term Selinger \& Bruinsma took the expression

$$
F_{\text {int }} / N k T \approx(N / V) L^{2} D\langle|\sin \gamma|\rangle \exp \left(-\left[2 \pi L N V^{-1}\left\langle|a|^{2}\right\rangle\right]^{-1}\right),
$$

which is equal to (51) in the nematic phase where the chains are orientationally but not spatially restricted so that $\left\langle|\boldsymbol{a}|^{2}\right\rangle=\infty$. The last factor represents a primitive expression for the decrease in excluded-volume interaction for spatially localized chains with finite $\left\langle|\boldsymbol{a}|^{2}\right\rangle$, like in an hexagonal phase. Both $\langle|\sin \gamma|\rangle$ and $\left\langle|\boldsymbol{a}|^{2}\right\rangle$ in (78) may be expressed in terms of $g(q)$, so that the total free energy (77) and (78) is a functional of $g(q)$.

Minimizing the free energy leads to the following general form of $g(q)$

$$
g(q)=\tau+\sigma q^{2}+\epsilon q^{4}
$$

with parameters $\tau$ and $\sigma$ which may take the following values:

(a) $\tau=\sigma=0$. The isotropic state without spatial or orientational order.

(b) $\tau=0, \sigma \neq 0$. The nematic state with strongly confined orientations but without spatial order $\left(\left\langle|\boldsymbol{a}|^{2}\right\rangle=\infty\right)$.

(c) $\tau \neq 0, \sigma \neq 0$. A spatially and orientationally confined state, possibly corresponding to an hexagonal state.

Phil. Trans. R. Soc. Lond. A (1993) 




Figure 5. Theoretical phase diagram of semi-flexible chains in terms of the volume fraction of chains and ratio of diameter and persistence length. Tie lines are shown in the two phase regions. (After Selinger \& Bruinsma 1991.)

Combining cases $(a)$ and $(b)$ with (75) (and applying $\left|\hat{\boldsymbol{u}}_{q}\right|=q\left|\boldsymbol{a}_{q}\right|$ ) leads to expression (69) if $\sigma$ is given in units $k T$, so that this more general method leads to Helfrich's assumption of mode suppression in the nematic state. Moreover, distribution function (76) combined with $g(q)=\sigma q^{2}+\epsilon q^{4}$ may be shown equivalent to a gaussian distribution (10) in $\theta$. Therefore, Selinger \& Bruinsma (1991) find an isotropicnematic phase transition at exactly the same concentrations as (55). For spatially confined chains the result found by this method would only be the same as Helfrich's (68) if $\sigma$ were 0 .

The total phase diagram found by Selinger \& Bruinsma is shown in figure 5 . Unfortunately, the hexagonal phase is only formed at a volume fraction above close packing of the chains, which reflects the inadequacy of a second virial theory to describe the hexagonal phase. However, choosing a more realistic form for the free energy could lead to better results.

In formulating such a theory for longer-ranged interactions (like electrostatic) one must be aware that fluctuations on a deflection length scale may exert a drastic influence on the effective potential. Odijk (1993) showed that for polyelectrolytes in an hexagonal state the electrostatic potential for simple rods has to be renormalized by a potentially large factor $\exp \left(\frac{1}{2} \kappa^{2} d^{2}\right)$ with $d$ the mean deviation of the chain from the hexagonal lattice points.

Finally, although beyond the scope of this qualitative overview, we want to call attention to the theories for nematic phases of wormlike chains of finite length, starting from Khokhlov \& Semenov (1982) and including several interpolating theories (Odijk 1986; Hentschke 1990; DuPré \& Yang 1991; Sato \& Teramoto 1990). For comparison with experiments this régime is obviously very important. Another important extension is the influence of charge (Odijk 1986; Vroege 1989) or bidispersity (Odijk 1986).

\section{Comparison with experiment}

Lyotropic liquid crystals occur in a wide variety of dispersions of rodlike colloidal particles and stiff macromolecules which can be purely synthetic or may be obtained from biological sources. For an understanding of the experimental results on lyotropic liquid crystal phase transitions the theories treated in the preceding sections provide a good framework. In general, however, before a quantitative 
comparison with experimental data can be made, a combination and/or extension of the theories discussed is needed.

Here we will focus the discussion on a number of systems representative for neutral and charged rigid rodlike colloids and semi-flexible macromolecules. As far as possible the systems selected are those with a well-defined size or molecular mass distribution and known molecular or particle parameters such as length, diameter, persistence length and charge density. Only for such systems can a meaningful comparison of experimental and theoretical results be made. The liquid crystal transition in a number of systems we will discuss later is in fact an isotropic-cholesteric transition. Assuming that the chiral part of the interparticle interaction that gives rise to a cholesteric rather than a nematic liquid crystal phase represents only a weak perturbation we will interpret the coexisting concentrations with theories that refer to the isotropic-nematic transition.

\section{(a) Neutral rigid rodlike colloidal particles}

It is only very recently that a stable system of uncharged rigid rodlike colloidal particles has been prepared: sterically stabilized boehmite rods dispersed in cyclohexane (Buining et al. 1992). For boehmite rods with an average length of about $200 \mathrm{~nm}$ and average aspect ratio of about 20, Buining (1992) found that the biphasic region lies between volume fractions $\phi_{i}=3.8 \%$ and $\phi_{n}=14.6 \%$. The concentrations of the coexisting isotropic and nematic phase change with the overall concentration. The rather low volume fraction $\phi_{i}$, the large width of the biphasic region and the observed variation of the concentrations of the coexisting phases are clearly related to the polydispersity of the boehmite particles. Representing the polydisperse system by a bidisperse system, the experimentally observed features of the isotropicnematic transition can be explained, at least qualitatively, with the simple theory outlined in $\S 3 a$. After several months a second, less concentrated, nematic phase is formed between the isotropic and the original nematic phase. This again may explained by the polydispersity of the particles assuming that the sample contains rods of sufficiently different lengths to exceed the minimum length ratio necessary for the appearance of a second nematic phase.

\section{(b) Charged rigid rodlike colloidal particles}

Liquid crystal phases have been observed in a variety of aqueous dispersions of charged rodlike colloidal particles. The most widely investigated and also the best characterized system in this category is tobacco mosaic virus (TMV). TMV is a cylindrical particle, consisting of a rigid protein shell enclosing double stranded RNA, with a length $L=300 \mathrm{~nm}$ and a diameter $D=18 \mathrm{~nm}$. The charge density of tobacco mosaic virus depends on $\mathrm{pH}$. Near neutral $\mathrm{pH}$ the charge density is believed to be $-1 \mathrm{e}$ to $-2 \mathrm{e}$ per protein subunit which corresponds to a linear charge density of $-7 \mathrm{e}$ to $-14 \mathrm{e}$ per nanometre. This seemingly large uncertainty has little effect on the calculations of the phase boundaries because the electrostatic interaction between two highly charged long rods is rather insensitive to the precise value of the linear charge density owing to the nonlinear screening effect of the counterions.

Recently, Fraden et al. (1989) measured the coexisting isotropic and nematic concentrations over a wide range of ionic strength (see figure 6 ). They found a strong ionic strength dependence of the isotropic-nematic phase boundaries, which clearly demonstrates that the electrostatic repulsion is an important factor in the phase behaviour of the system. The theoretical results obtained with the Onsager theory 
Figure 6

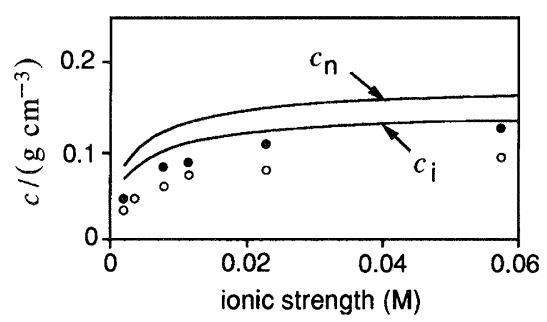

Figure 7



Figure 6. Comparison of the experimental concentrations of coexisting isotropic $(\circ)$ and nematic phases (•) of TMV dispersions as a function of ionic strength (Fraden et al. 1989) with theoretical results (solid curves, Sato \& Teramoto 1991).

Figure 7. Tentative phase diagram of TMV dispersions as a function of salt concentration (after Wen 1989).

including the effect of screened Coulomb interactions (see §4) lie considerably above the experimental values. This is not surprising as the hard-rod dimensions of TMV give a ratio $L / D \approx 17$, which is certainly not large enough for the Onsager approach to be quantitatively valid. Indeed Sato \& Teramoto (1991) going beyond the second virial approximation for the hard core part obtained much better agreement.

In 1950 Oster reported layerlike ordering in dispersions of TMV in the form of an 'iridescent' gel, which separated from a salt-free cholesteric phase. The periodicity of $340 \mathrm{~nm}$ determined by Oster is close to the TMV length of $300 \mathrm{~nm}$ which points to a structure formed by layers of TMV oriented perpendicular to the scattering planes. Similar results were found by Kreibig \& Wetter (1980) in an optical diffraction study of a number of different (tobacco) mosaic viruses. X-ray measurements by Fraden $e t$ al. (1982) showed that the iridescent phase of TMV in salt-free water is either truly colloidal crystalline or smectic B (with order within each layer but no registry of the ordering between consecutive layers). More recently, Wen et al. (1989) using a combination of optical and X-ray diffraction demonstrated that under conditions of high salt a smectic-A phase was formed, coexisting with a crystalline phase at higher volume fractions. In unbuffered solutions only a colloidal crystal was observed besides isotropic and nematic phases. This leads to the tentative phase diagram of figure 7 (after Wen 1989). Note that whereas one might interpret the effect of a decrease of the electrolyte concentration as a lowering of the effective $(L / D)$ ratio, the resulting phase diagram cannot be mapped on the hard spherocylinder diagram (Veerman \& Frenkel 1990). Lowering of the $(L / D)$ ratio in the latter case first results in the loss of stability of the nematic phase and only for still lower values of $(L / D)$ the smectic phase disappears. Apparently the effect of long-range electrostatic repulsion between the particles is a relative destabilization of the smectic phase with respect to the competing nematic and crystal phase.

\section{(c) Neutral semi-flexible polymers}

A well-characterized neutral semi-flexible polymer is Schizophyllan in water. Schizophyllan is a triple-helical polysaccharide with a diameter $D=1.7 \mathrm{~nm}$ and persistence length $P=200 \mathrm{~nm}$. The liquid crystal phase behaviour of Schizophyllan in water has been studied extensively by Teramoto and coworkers (Van \& Teramoto 1982; Itou \& Teramoto $1984 a, b$; Kojima et al. 1987) using samples with molecular masses varying from 65800 and 800000 which corresponds with contour lengths 
Figure 8



Figure 9

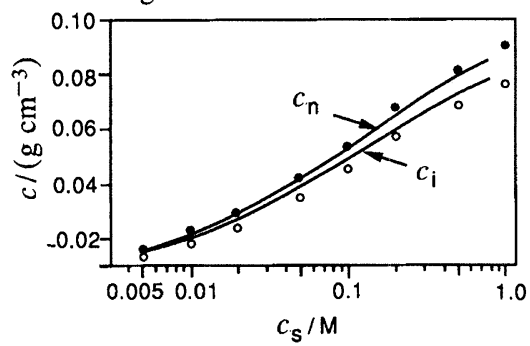

Figure 8. Comparison of the experimental concentrations of coexisting isotropic ( $\square$ ) and nematic (-) phases of aqueous solutions of Schizophyllan with different molecular weights (Itou \& Teramoto 1988) with theoretical results (dashed and solid curves, Odijk 1986). - - , $\phi_{n} P / D$; ,$--- \phi_{i} P / D$.

Figure 9. Comparison of experimental concentrations of coexisting isotropic (O) and nematic ( $)$ phases of aqueous solutions of Xanthane $\left(M=614 \times 10^{3}\right)$ as a function of salt concentration (Sato et al. 1990) with theoretical results (solid curves, Sato \& Teramoto 1991).

$L=30-370 \mathrm{~nm}$. This means that the number of persistence length units $N_{P}=L / P$ within each chain varies from 0.15 to 1.85 . This implies that neither the results for the rigid rod limit $(P \gg L \gg D)$ nor those for the semi-flexible limit $(L \gg P \gg D)$ are valid. In figure 8 we compare the measured isotropic-nematic (cholesteric) coexistence concentrations with the values calculated with the interpolating formulae for intermediate values of $N_{P}$ given by Odijk (1986). The overall agreement between theory and experiment is satisfactory, although the width of the biphasic gap is experimentally much larger than predicted by theory.

Teramoto and coworkers (Itou \& Teramoto 1984a, b, 1988; Kojima et al. 1987; Sato et al. 1989) have also studied bidisperse systems of Schizophyllan with different molecular mass ratios. Only for a molecular mass ratio as high as $12\left(M_{1}=68.500\right.$, $\left.M_{2}=800.000\right)$ there is a three-phase region in which an isotropic phase coexists with two nematic (cholesteric) phases as well as a biphasic region where two nematic (cholesteric) phases coexist. As we saw in $\S 3 a$ this kind of behaviour has been predicted for bidisperse systems of rigid rods when the length ratio exceeds 3.2. From the experimental data it is clear that in the case of wormlike chains the minimum length ratio for the occurrence of two nematic phases is considerably higher. This is supported by recent theoretical results of Teramoto (personal communication).

\section{(d) Semi-flexible polyelectrolytes}

Polyelectrolytes show good solubility in water. These systems can therefore be studied over an extensive concentration range. Again the limitation is that for a meaningful comparison with theory the systems must be monodisperse and - in addition to the usual parameters $L, D$ and $P$ - the (linear) charge density $\nu$ of the 
polyelectrolyte and the salt concentration in water must be known. A well characterized stiff polyelectrolyte is Xanthan. Under certain conditions Xanthan is a double-helical polysaccharide with a diameter $D=2.2 \mathrm{~nm}$, a persistence length $P=120 \mathrm{~nm}$ and a linear charge density of $-3 \mathrm{e}$ per nanometre. The liquid crystal phase behaviour of Xanthan in water has again been studied extensively by Teramoto and coworkers (Sato et al. 1990; Inatomi et al. 1992) using samples with molecular mass varying from 110.000 to $980.000(L=55-500 \mathrm{~nm})$ and salt concentrations between $0.005 \mathrm{~m}$ and $1 \mathrm{~m}$. The experimental data on these systems have been analysed by Sato \& Teramoto (1991) using the theory of Stroobants et al. (1986), discussed in $\S 4$, as modified to wormlike chains by Odijk (1986) and incorporating end effects in the electrostatic interaction. The agreement between theory and experiment is quite good (figure 9).

In addition to a nematic liquid crystal phase a columnar liquid crystal phase has been reported for Xanthan (Livolant \& Bouligand 1986).

\section{Concluding remarks}

After nearly 50 years Onsager's explanation of the formation of a nematic phase - a loss of orientational entropy compensated by a gain in free volume - remains unassailed, while its extension by Khokhlov \& Semenov to semi-flexible chains by inclusion of the configurational entropy has considerably widened the range of the theory in terms of applicability to experiments. Looking back upon the preceding section we may conclude that there exists a considerable amount of experimental material on well-characterized systems which can be described (semi) quantitatively with the Onsager theory and its extensions.

Although there are a number of theoretical issues regarding the isotropic-nematic phase transition which require further attention, notably the role of attractive forces, it seems fair to say that the main challenges are offered by the more highly ordered phases, such as the smectic and hexagonal phase. For hard core systems the trend was set by computer simulations followed by density functional theory. However, for charged rodlike particles no such results are available. The observed phase behaviour of TMV which differs qualitatively from hard rods certainly warrants such efforts.

Another question which deserves attention is why rigid rodlike particles undergo nematic-smectic phase transitions, whereas semi-flexible chains show nematichexagonal phase transitions. The theory of Selinger \& Bruinsma (1991) indicates that infinitely long semi-flexible chains indeed undergo a nematic-hexagonal phase transition, albeit at an unphysical density (larger than close packing). However, it would be interesting to have a theory that can handle short semi-flexible chains (say $L / P$ around 1 ) with or without electrostatic interactions as well. Only then can we hope to understand the rich but complicated phase behaviour that has been observed in concentrated solutions of persistence length fragments of DNA (Rill et al. 1983, 1991 ; Rill 1986; Strzelecka \& Rill 1987, 1990; Strzelecka et al. 1988; Livolant et al. 1989 ; Durand et al. 1992).

We thank Ms Marina Uit de Bulten and Ms Toni Vos for their accurate preparation of the typescript and Mr Jan den Boesterd for preparing the figures. 


\section{References}

Allen, M. P., Frenkel, D. \& Talbot, J. 1989 Comput. Phys. Rep. 9, 301.

Birshtein, T. M., Kolegov, B. I. \& Pryamitsyn, V. A. 1988 Vysokomol. Soyed. A 30, 348 (1988 Polym. Sci. USSR 30, 316).

Brenner, S. L. \& Parsegian, V. A. 1974 Biophys. J. 14, 327.

Buining, P. A., Veldhuizen, Y. S. J., Pathmamanoharan, C. \& Lekkerkerker, H. N. W. 1992 Colloids Surf. 64, 47.

Buining, P. A. 1992 Proefschrift. University of Utrecht.

Cotter, M. A. 1977 J. chem. Phys. 66, 1098.

Deutsch, J. M. \& Goldenfeld, N. D. $1982 a$ J. Phys. A 15, L71.

Deutsch, J. M. \& Goldenfeld, N. D. $1982 b$ J. Phys. 43, 651.

Dijkstra, M., Frenkel, D. \& Lekkerkerker, H. N. W. 1993 Physica A 193, 374

DuPré, D. B. \& Yang, S. 1991 J. chem. Phys. 94, 7466.

Durand, D., Doucet, J. \& Livolant, F. 1992 J. Phys. II 2, 1769.

Fixman, M. \& Skolnick, J. 1978 Macromolecules 11, 863.

Flory, P. J. 1956 Proc. R. Soc. Lond. A 234, 60, 73.

Fraden, S., Caspar, D. L. D. \& Phillips, W. C. 1982 Biophys. J. 37, 94a.

Fraden, S., Maret, G., Caspar, D. L. D. \& Meyer, R. B. 1989 Phys. Rev. Lett. 63, 2068.

Frenkel, D. 1987 a J. phys. Chem. 91, 4912 (erratum: 1988, 92, 5314).

Frenkel, D. 1987 b Mol. Phys. 60, 1.

Frenkel, D. 1988 J. phys. Chem. 92, 3280 (erratum: 1988, 92, 5314).

Frenkel, D. 1989 Liquid Cryst. 5, 929.

Frenkel, D., Mulder, B. M. \& McTague, J. P. 1984 Phys. Rev. Lett. 52, 287.

Frenkel, D. \& Mulder, B. M. 1985 Mol. Phys. 55, 1171.

Frenkel, D., Lekkerkerker, H. N. W. \& Stroobants, A. 1988 Nature, Lond. 332, 822.

Gelbart, W. M. \& Baron, B. A. 1977 J. chem. Phys. 66, 207.

Helfrich, W. 1978 Z. Naturforsch. A 33, 305.

Helfrich, W. \& Harbich, W. 1985 Chem. Scr. 25, 32.

Hentschke, R. 1990 Macromolecules 23, 1192.

Inatomi, S., Jinbo, Y., Sato, T. \& Teramoto, A. 1992 Macromolecules 25, 5013.

Itou, T. \& Teramoto, A. 1984 a Macromolecules 17, 1419.

Itou, T. \& Teramoto, A. $1984 b$ Polym. J. 16, 779.

Itou, T. \& Teramoto, A. 1988 Macromolecules 21, 2225.

Khokhlov, A. R. \& Semenov, A. N. 1981 Physica A 108, 546.

Khokhlov, A. R. \& Semenov, A. N. 1982 Physica A 112, 605.

Khokhlov, A. R. \& Semenov, A. N. 1985 J. statist. Phys. 38, 161.

Kojima, T., Itou, T. \& Teramoto, A. 1987 Polymer J. 19, 1225.

Kratky, O. \& Porod, G. 1949 Rec. Trav. Chim. 68, 1106.

Kreibig, U. \& Wetter, C. 1980 Z. Naturforsch. C 35, 750.

Lekkerkerker, H. N. W., Coulon, P., van der Haegen, R. \& Deblieck, R. 1984 J. chem. Phys. 80, 3427.

Lifshitz, I. M. 1968 Z. Eksp. Teor. Fiz. 55, 2408 (1969 Sov. Phys. JETP 28, 1280).

Lifshitz, I. M., Grosberg, A. Yu \& Khokhlov, A. R. 1978 Rev. mod. Phys. 50, 683.

Livolant, F. \& Bouligand, Y. 1986 J. Phys. 47, 1813.

Livolant, F., Levelut, A. M., Doucet, J. \& Benoit, J. P. 1989 Nature, Lond. 339, 724.

Mulder, B. M. 1987 Phys. Rev. A 35, 3095.

Nyrkova, I. A. \& Khokhlov, A. R. 1986 Biofyzika 31, 771 (1986 Biophys. 31, 839).

Odijk, T. 1983 Macromolecules 16, 1340.

Odijk, T. 1985 Polym. Commun. 26, 197.

Phil. Trans. R. Soc. Lond. A (1993) 
Odijk, T. 1986 Macromolecules 19, 2313.

Odijk, T. 1988 Integration of fundamental polymer science and technology, vol. 2 (ed. P. J. Lemstra $\&$ L. A. Kleintjes). London: Elsevier.

Odijk, T. 1993 Biophys. Chem. 46, 69.

Odijk, T. \& Lekkerkerker, H. N. W. 1985 J. phys. Chem. 89, 2090.

Onsager, L. 1942 Phys. Rev. 62, 558.

Onsager, L. 1949 Ann. NY Acad. Sci. 51, 627.

Oster, G. 1950 J. Gen. Physiol. 33, 445.

Parsons, J. D. 1979 Phys. Rev. A 19, 1225.

Rill, R. L. 1986 Proc. natn. Acad. Sci. U.S.A. 83, 342.

Rill, R. L., Hilliard, P. R. \& Levy, G. C. 1983 J. Biol. Chem. 258, 250.

Rill, R. L., Strzelecka, T. E., Davidson, M. W. \& Van Winkle, D. H. 1991 Physica A 176, 87.

Sato, T. \& Teramoto, A. 1990 Mol. Cryst. Liquid Cryst. 178, 143.

Sato, T. \& Teramoto, A. 1991 Physica A 176, 72.

Sato, T., Ikeda, N., Itou, T. \& Teramoto, A. 1989 Polymer 30, 311.

Sato, T., Kakihara, T. \& Teramoto, A. 1990 Polymer 31, 824.

Selinger, J. V. \& Bruinsma, R. F. 1991 Phys. Rev. A 43, 2922.

Sluckin, T. J. 1989 Liquid Cryst. 6, 111.

Stigter, D. 1977 Biopolymers 16, 1435.

Straley, J. P. 1973 Mol. Cryst. Liquid Cryst. 24, 7.

Stroobants, A., Lekkerkerker, H. N. W. \& Odijk, T. 1986 Macromolecules 19, 2232.

Stroobants, A. 1992 Phys. Rev. Lett. 69, 2388.

Strzelecka, T. E. \& Rill, R. L. 1987 J. Am. chem. Soc. 109, 4513.

Strzelecka, T. E. \& Rill, R. L. 1990 Biopolymers 30, 57.

Strzelecka, T. E., Davidson, M. W. \& Rill, R. L. 1988 Nature, Lond. 331, 457.

Tjipto-Margo, B. \& Evans, G. T. 1990 J. chem. Phys. 93, 4254.

Van, K. \& Teramoto, A. 1982 Polymer J. 14, 999.

Van der Schoot, P. P. A. M. \& Odijk, T. 1992 J. chem. Phys. 97, 515.

Veerman, J. A. C. \& Frenkel, D. 1990 Phys. Rev. A 41, 3237.

Vroege, G. J. \& Odijk, T. 1988 Macromolecules 21, 2848.

Vroege, G. J. 1989 J. chem. Phys. 90, 4560.

Vroege, G. J. \& Lekkerkerker, H. N. W. 1992 Rep. Prog. Phys. 55, 1241.

Vroege, G. J. \& Lekkerkerker, H. N. W. 1993 J. Phys. Chem. 97, 3601.

Wen, X., Meyer, R. B. \& Caspar, D. L. D. 1989 Phys. Rev. Lett. 63, 2760.

Wen, X. 1989 Ph.D. thesis, Brandeis University.

Yamakawa, H. 1971 Modern theory of polymer solutions. New York: Harper and Row.

Yamakawa, H. 1984 A. Rev. Phys. Chem. 35, 23. 